\title{
Birth Trauma and Post Traumatic Stress Disorder (PTSD)
}

\section{Morgan Staver BSN, RN, PMH-C}

The National Perinatal Association (NPA)is an interdisciplinary organization that strives to be a leading voice for perinatal care in the United States. Our diverse membership is comprised of healthcare providers, parents \& caregivers, educators, and service providers, all driven by their desire to give voice to and support babies and families at risk across the country.

Members of the NPA write a regular peer-reviewed column in Neonatology Today.

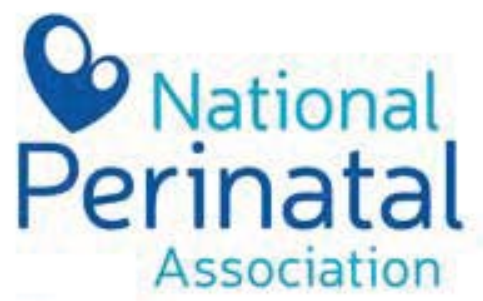

Educate. Advocate. Integrate.

\section{"The recognition of birth}

trauma is a relatively

new phenomenon, first

referenced in the scientific

literature in the 1990s. (1)"

\section{What is birth trauma?}

The recognition of birth trauma is a relatively new phenomenon, first referenced in the scientific literature in the 1990s. (1)

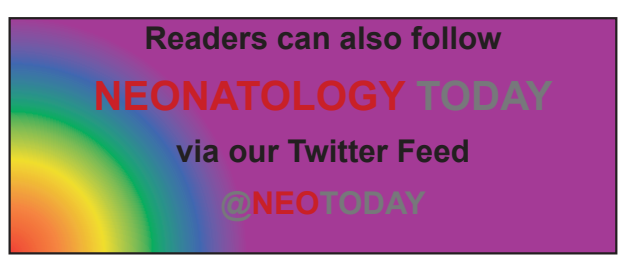

Birth trauma is a subjective experience: it is always in the eye of the beholder. (2) While we as clinicians cannot diagnose a birth as traumatic, we can recognize its place in obstetric, neonatal, and psychiatric care. Birth trauma is the perception of actual or threatened serious harm to the mother or the infant during any phase of childbirth. (2) The phenomenon of birth trauma is related to criterion $A$ for posttraumatic stress disorder (PTSD): exposure to actual or threatened death, serious injury, or sexual violence through a direct experience, witnessing the event, learning that the event has happened to someone close, or experiencing repeated or extreme exposure to details of a traumatic event. (3) The existence of birth trauma and its potential consequences have been historically under-recognized by professionals and the public because of the societal belief that childbirth should always be a positive experience. (4) In reality, the birth process can be dangerous and requires a woman to put her trust in a medical team that likely includes individuals that the woman has never met. $45 \%$ of women who give birth perceive their birth as a traumatic event. (5)

Women who have experienced a traumatic birth describe an array of events that turned the birth from a positive experience into a traumatic one (see Table 1 for a nonexhaustive list of traumatic birth events). $(2,6)$ The potentially traumatic events during childbirth outlined can result in infant complications and admission to a neonatal intensive care unit (NICU). The NICU environment carries with it additional potential traumatic events. A non-exhaustive list of potential NICU-specific traumatic events includes medical intervention at birth, unexpected or prolonged NICU hospitalization, immediate separation from newborn, seeing the infant in the NICU for the first time, code blue events, failed extubations, the NICU waiting room, or surgeries. (7)

No matter the reason that the birth is perceived as traumatic, traumatic birth is a complicated phenomenon. Women who have experienced a traumatic birth have described the following essential themes of birth trauma: perception of lack of caring from those who are supposed to care (feeling abandoned, stripped of dignity, lack of support or reassurance), poor communication from key players, feeling invisible, feeling powerless (betrayals of trust, not feeling protected by staff), and wondering if the ends justify the means (i.e., pondering if having a healthy baby justifies the endured trauma and experiencing guilt associated with that thought). (2)

\begin{tabular}{|l|}
\hline Table 1. Traumatic Birth Events \\
\hline Stillbirth or infant death \\
\hline Emergency cesarian delivery \\
\hline Fetal distress \\
\hline Cardiac arrest \\
\hline Inadequate medical care \\
\hline Fear of epidural \\
\hline Congenital anomalies \\
\hline Inadequate pain relief \\
\hline Postpartum hemorrhage \\
\hline Manual removal of placenta \\
\hline Use of forceps \\
\hline Vacuum extraction \\
\hline Skull fracture \\
\hline Severe toxemia \\
\hline Premature birth \\
\hline Prolonged or painful labor \\
\hline Rapid delivery \\
\hline Degrading experiences \\
\hline Severe pre-eclampsia \\
\hline $3^{\text {rd }}$ or $4^{\text {th }}$-degree laceration \\
\hline Hyperemesis gravidarum \\
\hline Shoulder dystocia \\
\hline
\end{tabular}

\section{Who experiences birth trauma?}

A traumatic birth can happen to anyone: it is independent of race or socioeconomic status. (8) The most important factors regarding how a woman conceptualizes her delivery experience are a) the support she receives from caregivers, b) her involvement in decision-making processes, and c) her expectations of the birth experience and if they were met. (4) Some women are more likely than others to perceive their birth as traumatic due to their previous life experiences. Women with a history of psychological problems, trauma or sexual abuse, anxiety, or nulliparity are more likely than women with none of these experiences to perceive birth as a traumatic 
event. (9)

Perceiving birth as traumatic does not always lead to the development of PTSD. Risk factors present before birth (i.e., before or during pregnancy) and during birth influence if a woman who experiences a traumatic birth will develop symptoms of PTSD and how quickly the PTSD symptoms may resolve (see Table 2 for a list of risk factors). $(8,9)$

PTSD does not occur in all populations equally. In a recent metaanalysis, researchers estimated that the community prevalence of postpartum PTSD is $3.1 \%$. In contrast, the prevalence in the atrisk population (i.e., perinatal risk, trauma history, psychiatric history) is estimated to be $15.7 \%$. (8) In women who develop PTSD after birth, postpartum depression, maternal complications, infant complications, trauma history, negative interactions with staff,(8) poor coping, and stress are all correlated with PTSD symptoms. (9)

\section{"If all PTSD diagnostic criteria are met, but the symptoms do not last for more than one month, a diagnosis of acute stress disorder is more appropriate. (3)."}

\section{What is the relationship between birth trauma and PTSD?}

Not everyone who experiences a traumatic birth will go on to develop PTSD. While $45 \%$ of women state that their birth was traumatic, only around $3 \%$ of women develop clinically significant PTSD. (5) As described above, birth trauma fulfills criterion A, exposure to a traumatic event, to diagnose PTSD. Other diagnostic criteria for PTSD broadly include symptoms of re-experiencing, avoidance, negative changes in cognition or mood, and hyperarousal. These symptoms must last for at least one month, cause clinically significant impairment in functioning, and cannot be attributable to another medical condition or substance use. If all PTSD diagnostic criteria are met, but the symptoms do not last for more than one month, a diagnosis of acute stress disorder is more appropriate. (3) It is possible to experience PTSD or acute stress disorder symptoms after a traumatic birth and not meet full diagnostic criteria for either disorder.
PTSD in the postpartum period can occur due to birth trauma. However, it can also present as a result of other traumatic events in the mother's lifetime that put her at risk for PTSD after childbirth (e.g., history of domestic violence or sexual assault). (8) Birth experiences and trauma history interact and put a woman at higher risk for developing PTSD after birth. (9)

\section{How is postpartum PTSD different from 'general' PTSD?}

While postpartum PTSD meets all the DSM 5 criteria for PTSD, unique factors make it distinct from PTSD resulting from another traumatic event. For instance, childbirth may be the only criterion A event for a PTSD diagnosis that is generally assumed to be a joyous event. Experiencing a seemingly positive life event such as childbirth as traumatic may affect how a woman conceptualizes the legitimacy of the traumatic experience and may influence reporting of symptoms. The symptom presentation of postpartum PTSD shows a unique clustered pattern:

- $\quad$ Re-experiencing and avoidance related to childbirth, with the physical sequelae of birth and the baby acting as a constant reminder of the traumatic birth, and

- $\quad$ Symptoms of arousal and negative alterations in cognition or mood that align with the general PTSD criteria

The social implications resulting from a traumatic birth also affect symptom presentation of postpartum PTSD uniquely from 'general' PTSD. The effects of the traumatic birth are experienced by and affect the whole family. A traumatic birth and postpartum PTSD are unique because biologic women exclusively experience them; the woman-only survivor profile's clinical, social, and political implications may play a role in the survivor's experiences after a traumatic birth.(10)

\section{Why does birth trauma matter?}

Although most women who experience a traumatic birth do not develop PTSD, the core life experience of childbirth has been corrupted. If a woman develops PTSD after a traumatic birth, she will face potential consequences related to her healthcare and her family and baby's functioning. Documented behavioral consequences of PTSD due to birth trauma include avoidance of postpartum care, impaired parental-infant bonding, PTSD in a partner who witnessed the birth, sexual dysfunction, avoidance of future pregnancies, elective cesarean births in future pregnancies, difficulties with breastfeeding, yearly anniversary of traumatic birth, low self-esteem, lower birth weight infants, and lower breastfeed-

Table 2. Risk Factors Associated with PTSD After Childbirth

Pre-Birth Factors

Pregnancy psychopathology or depression during pregnancy

Fear of giving birth

Health complications during pregnancy

Previous counseling for pregnancy or birth problems

History of PTSD

Birth Factors

Subjective distress during labor

Negative feelings during birth

Lack of control or agency during birth

Obstetric emergencies

Operative births

Lack of support from staff

Dissociation during birth 
ing rates. $(4,6)$ Potential links also exist between maternal PTSD and infant cortisol, sleeping, and eating problems. There is mixed evidence for the associations between postpartum PTSD and preterm birth, fetal growth, head circumference, mother-infant bonding, and infant development. (11)

Obstetric and neonatal clinicians also should recognize that a traumatic birth experience has a complicated relationship with breastfeeding. A traumatic birth can impede breastfeeding by producing feelings of violation, physical pain, and detachment from the infant. Women who experienced a traumatic birth may perceive a relationship between the trauma and decreased milk supply. Breastfeeding may also produce intrusive flashbacks in some women. However, a traumatic birth can promote breastfeeding in some women because it may be a way for the mother to 'redeem' herself after a traumatic birth or provide an opportunity for grounding, a type of coping mechanism. The reasons why traumatic birth can be a motivating factor for some women but impede breastfeeding in other women are currently unknown. (12)

\section{What should neonatal care providers know about birth trau- ma?}

Obstetric and neonatal clinicians may encounter women who have experienced a traumatic birth more often than they realize. Because seemingly 'normal' births may be perceived as traumatic, it is vital for professionals working in this area to ask the mother about her thoughts and feelings about her birth experience. Pay special attention to women whose birth experiences included observable risk factors for a traumatic birth associated with developing PTSD (e.g., operative births or obstetric emergencies). (9) Clinicians should consider assessing women during pregnancy for the factors associated with an increased risk of developing PTSD after birth (e.g., depression during pregnancy, fear of childbirth, poor health or pregnancy complications, and a history of PTSD). (9)

If a patient has experienced traumatic birth or is at risk for developing PTSD, refer her for mental health treatment by a clinician that specializes in perinatal mental health, if possible. There are many effective treatments for PTSD, and the mental health professional will work with the patient to identify a treatment that best fits her needs. Potential treatments include psychotherapy and pharmacotherapy. Psychotherapy may be more likely to lead to a more significant reduction in PTSD symptoms that last longer than using first-line medications such as selective serotonin reuptake inhibitors alone. Trauma-focused psychotherapies effective for PTSD treatment are prolonged exposure therapy, cognitive processing therapy, and eye movement desensitization and reprocessing. (13) Currently, there is no evidence addressing primary prevention of birth trauma. However, expressive writing soon after delivery of a healthy infant may be an effective intervention to prevent the development of PTSD symptoms after childbirth. (4)

Obstetric clinicians should promote factors that have shown to be protective against traumatic birth. The factor with the most empirical support that may reduce a woman's chance of experiencing a traumatic birth or reduce her likelihood of developing postpartum PTSD is social support during birth. (9) Allowing the presence of a support person during birth is critical to reducing the risk for birth trauma and PTSD. Perceived lack of staff support and negative interactions with staff during delivery are modifiable risk factors for birth trauma and subsequent PTSD that obstetric and neonatal clinicians can target to reduce adverse outcomes.

\section{Conclusion}

Just under half of the women who give birth perceive their birth experience as traumatic, and a small but significant percentage of this population develops PTSD. The symptoms and experiences of women who experience traumatic birth are important to evaluate because birth trauma can go unrecognized if not assessed. Clinicians who work with this population should be aware of the risk factors for experiencing birth as traumatic, the risks for developing PTSD after birth, and the potential consequences of postpartum PTSD. Bringing a conversation about birth trauma to our patients can decrease any stigma attached to the experience and potentially identify more women who would benefit from psychiatric care.

\section{"Clinicians who work with this population should be aware of the risk factors for experiencing birth as traumatic, the risks for developing PTSD after birth, and the potential consequences of postpartum PTSD. Bringing a conversation about birth trauma to our patients can decrease any stigma attached to the experience and potentially identify more women who would benefit from psychiatric care. "}

\section{References:}

1. Goldbeck-Wood, S. Post-traumatic stress disorder may follow childbirth. BMJ. 1996;313(7060). doi:10.1136/ bmj.313.7060.774

2. Beck CT. Birth trauma: in the eye of the beholder. Nurs Res. 2004;53(1):28-35.

3. American Psychiatric Association. Diagnostic and Statistical Manual of Mental Disorders. Fifth Edition. American Psychiatric Association; 2013. doi:10.1176/appi. books.9780890425596

4. Graaff LF de, Honig A, Pampus MG van, Stramrood CAI. Preventing posttraumatic stress disorder following childbirth and traumatic birth experiences: a systematic review. Acta Obstet Gynecol Scand. 2018;97(6):648-656. doi:https://doi. org/10.1111/aogs. 13291

5. Adriana Lozada, Cheryl Tatano Beck. Childbirth PTSD.; 2017. https://birthful.com/podcastinfo/

6. Postpartum Support International. Perinatal Post Traumatic Stress Disorder. Presented at the: Perinatal Mood Disorders: Components of Care; 2020. https://www.postpartum. net

7. Postpartum Support International. Additional Risks: Special Populations. Presented at the: Perinatal Mood Disorders: Components of Care; 2020. www.postpartum.net

8. Grekin R, O'Hara MW. Prevalence and risk factors of postpartum posttraumatic stress disorder: A meta-analysis. Clin Psychol Rev. 2014;34(5):389-401. doi:10.1016/j. cpr.2014.05.003

9. Ayers S, Bond R, Bertullies S, Wijma K. The aetiology of posttraumatic stress following childbirth: a meta-analysis and theoretical framework. Psychol Med. 2016;46(6):11211134. doi:10.1017/S0033291715002706

10. Horesh D, Garthus-Niegel S, Horsch A. Childbirth-related 
PTSD: is it a unique posttraumatic disorder? J Reprod Infant Psychol. 2021;39(3):221-224. doi:10.1080/02646838.2021. 1930739

11. Cook N, Ayers S, Horsch A. Maternal posttraumatic stress disorder during the perinatal period and child outcomes: $A$ systematic review. J Affect Disord. 2018;225(Journal Article):18-31. doi:10.1016/j.jad.2017.07.045

12. Beck CT, Watson S. Impact of Birth Trauma on Breast-feeding: A Tale of Two Pathways. Nurs Res. 2008;57(4):228-236. doi:10.1097/01.NNR.0000313494.87282.90

13. Watts BV, Schnurr PP, Mayo L, Young-Xu Y, Weeks WB, Friedman MJ. Meta-Analysis of the Efficacy of Treatments for Posttraumatic Stress Disorder. J Clin Psychiatry. 2013;74(06):e541-e550. doi:10.4088/JCP.12r08225

Disclosures: The author does not have any conflicts to disclose.

Disclosure: The National Perinatal Association www.nationalperinatal.org is a 501 c 3 organization that provides education and advocacy around issues affecting the health of mothers, babies, and families.

NT

Corresponding Author:

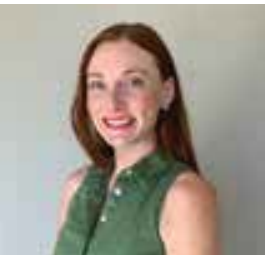

Morgan Staver BSN, RN, PMH-C

PhD-PMHNP Student

University of Nebraska Medical Center College of Nursing

Omaha, NE

Email:morgan.staver@unmc.edu

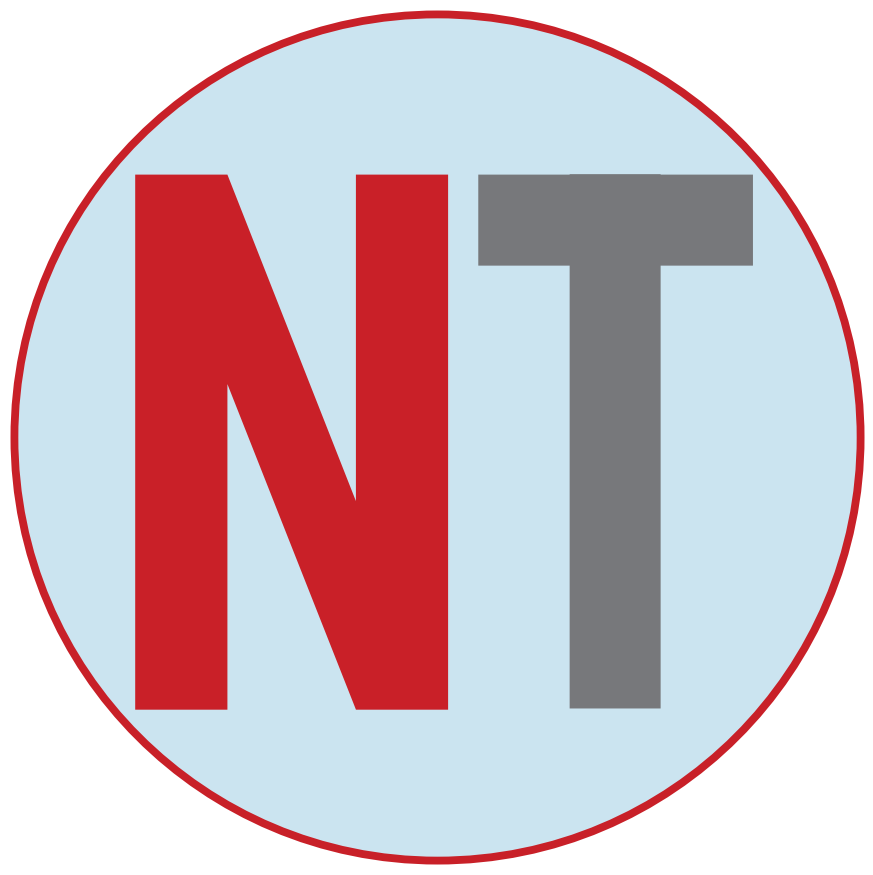

COPING WITH

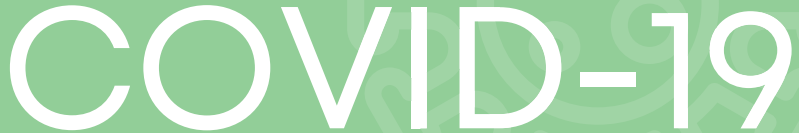

KEEP PATIENTS

UP-TO-DATE WITH

CHANGES IN

POLICIES SO THEY

KNOW WHAT TO

EXPECT. LISTEN TO

THEIR CONCERNS.
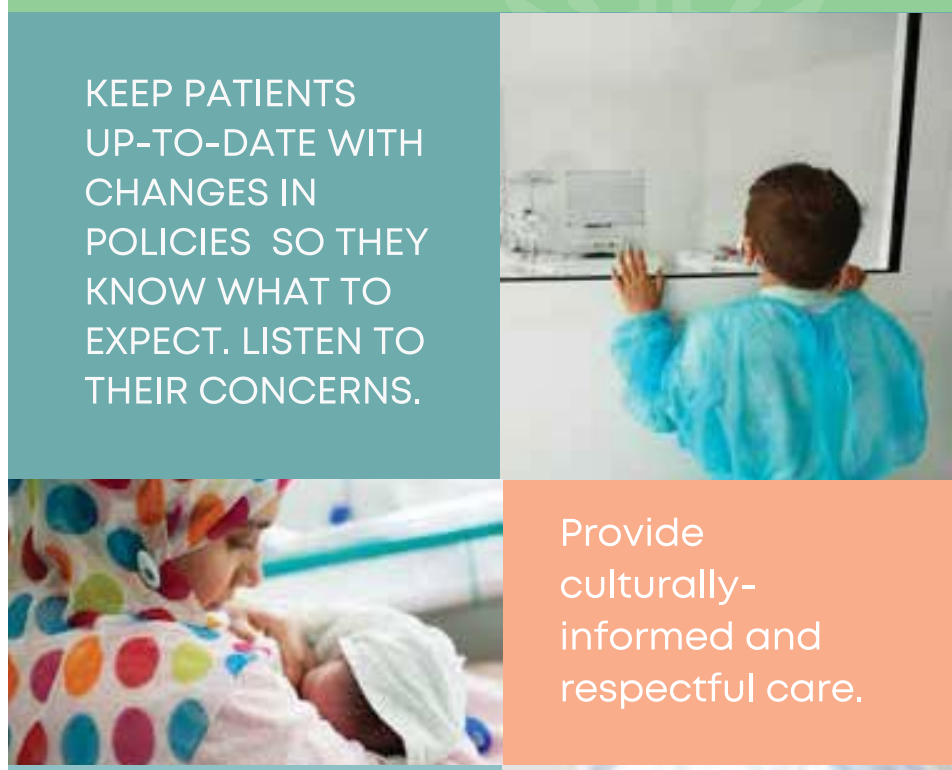

Provide

culturally-

informed and

respectful care.

TELL PARENTS

HOW YOU WILL

KEEP THEM AND

THEIR BABIES

SAFE DURING

THEIR NICU STAY.

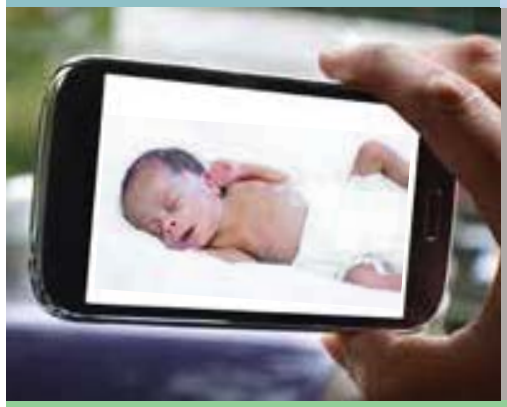

Use technology

like video chat

apps to include

family members

who can't visit

the NICU.

\section{myNICUnetwork.org}

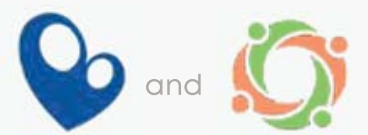

National Perinatal Association NICU Parent Network
My Perinatal Network and My NICU Network are products of a collaboration 